\title{
Translational research combining orthologous genes and human diseases with the OGOLOD dataset
}

\author{
José Antonio Miñarro-Giménez ${ }^{\mathrm{a}, *}$, Mikel Egaña Aranguren ${ }^{\mathrm{b}, \mathrm{d}}$ Boris Villazón-Terrazas ${ }^{\mathrm{c}, \mathrm{d}}$ \\ Jesualdo Tomás Fernández Breis ${ }^{\text {a }}$

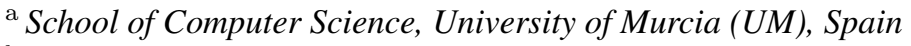 \\ ${ }^{\mathrm{b}}$ Biological Informatics, Centre for Plant Biotechnology and Genomics (CBGP), Technical University of Madrid \\ (UPM), Spain \\ ${ }^{\mathrm{c}}$ iSOCO, Avda. Partenon. 16-18, 28042, Madrid, Spain \\ ${ }^{\mathrm{d}}$ Ontology Engineering Group (OEG), School of Computer Science, Technical University of Madrid (UPM), Spain
}

\begin{abstract}
OGOLOD is a Linked Open Data dataset derived from different biomedical resources by an automated pipeline, using a tailored ontology as a scaffold. The key contribution of OGOLOD is that it links, in new RDF triples, genetic human diseases and orthologous genes, paving the way for a more efficient translational biomedical research exploiting the Linked Open Data cloud.
\end{abstract}

Keywords: Linked Open Data, Linked Data, orthologous genes, human genetic disease, translational research, biomedicine, RDF, SPARQL, OWL, ontology

\section{Introduction}

OGOLOD is a Linked Open Data (LOD) dataset that represents combined information about orthologous genes and human genetic diseases (Table 1). Orthologous genes present similarity due to the fact that they descend from a common ancestor [4]. Such genes can be used to extrapolate information from one organism to another; hence the interest of using them to infer new hypotheses about human genetic diseases, that is, understanding the genetic causes of diseases $[1,6]$. The growing interest for facilitating an efficient access to information about orthologous genes is also demonstrated by efforts like Quest for Orthologs [4].

OGOLOD is the output of an automated pipeline that obtains and adapts information from the following sources:

\footnotetext{
*Corresponding author. E-mail: jose.minya@ gmail.com
}

- Databases of orthologous genes: COG [15], Inparanoid [11], Homologene ${ }^{1}$, and OrthoMCL [3].

- OMIM$^{2}$ (Online Mendelian Inheritance in Man), a database holding information about human genetic diseases.

The information from these sources is collected as RDF instances using the OGO ontology (Figure 1). Thus, the OGO ontology provides the backbone in which to embed all the data collected from the sources: an axiomatised representation of the knowledge domain of orthologous genes and human genetic diseases. The OGO ontology combines widely used vocabularies from the life sciences domain, in doing so enhancing the capability of the OGOLOD dataset of being integrated with the rest of the (life sciences) LOD cloud. Such reused vocabularies describe different domains of interest: GO (gene product function,

\footnotetext{
${ }^{1}$ http://www.ncbi.nlm.nih.gov/homologene

${ }^{2}$ http://www.ncbi.nlm.nih.gov/omim/
} 
location, or process) [5], $\mathrm{ECO}^{3}$ (evidence code types that support the link of a gene product with a GO term), NCBI taxonomy (classification of organisms) [13], RO (a set of widely used biological relations) [14], and HPO (a vocabulary for human phenotypic abnormalities) [12]. OGOLOD has also links to other LOD datasets, providing a unique entry point to combine information about orthologous genes with other extant LOD datasets.

OGOLOD allows researchers to combine information about the evolutionary relationship of genes, their biological implications and their relation with disorders in their research hypotheses. For instance, researchers of genetic disorders could find genes in other species with similar function to the ones associated with such disorders. As a consequence of such integration of information, OGOLOD gives the researchers the ability to answer queries like "diseases in which the orthologous genes of gene X are involved" (Table 2), or "all orthologous genes that belong to Rattus Norvegicus and are related to the genes involved in lung cancer" (Table 4).

OGOLOD is the LOD version of the OGO Knowledge Base (KB) $[8,9]$ ("OGOLOD" stands for "OGO Linked Open Data"). OGOLOD has been produced by following the methodological guidelines described in [16] for publishing LOD datasets, committing to an iterative and incremental life cycle model. The process of converting OGO to OGOLOD and the resulting OGOLOD dataset are detailed in [10]; this paper focuses on the OGOLOD dataset alone, providing a self-contained and brief overview.

\section{Discussion}

\subsection{URI design}

The URI design in OGOLOD is based on slash URIs, instead of hash URIs, since the dataset has a considerable size. Also, the URIs reflect the structure of the model, making a distinction between classes and instances, to improve the usability of the dataset. ${ }^{4}$

\footnotetext{
${ }^{3}$ http://www. obofoundry.org/cgi-bin/detail. cgi?id=evidencecode

${ }^{4}$ e.g., the URI for the class Gene is http://miuras. inf.um.es/ogolod/ontology/Gene, and the URI for the Gene 67440 is http://miuras.inf.um.es/ogolod/ resource/Gene/67440: note the distinction between ontology and resource.
}

Table 1

Basic information and metrics of the OGOLOD dataset. In the case of outbound links, the <owl : sameAs> relationships were established manually, by adding a relation when the original database ID was the same in both datasets.

\begin{tabular}{|c|c|}
\hline Name & OGOLOD \\
\hline Website & http://miuras.inf.um.es/ ogo/ogolod.html \\
\hline SPARQL endpoint & http://miuras.inf.um.es/sparql \\
\hline Datahub entry & http://thedatahub.org/dataset/ogolod \\
\hline RDF dumps & http://miuras.inf.um.es/dump/ogolod.zip \\
\hline Logs & http://miuras.inf.um.es/sparqlLogs/query.zip \\
\hline Sitemap & $\begin{array}{l}\text { http://miuras.inf.um.es/sitemap_index.xml } \\
\text { (It is being indexed at http://sindice.com/) }\end{array}$ \\
\hline Version $(2012 / 5 / 10)$ & 0.1 \\
\hline Number of triples & $38,035,102$ \\
\hline $\begin{array}{l}\text { Number of links to } \\
\text { bio2rdf-chebi }\end{array}$ & $3,456,570$ \\
\hline $\begin{array}{l}\text { Number of links to } \\
\text { bio2rdf-omim }\end{array}$ & 18,141 \\
\hline Model expressivity & $\mathcal{A L C}(\mathcal{D})$ \\
\hline License & CC0 1.0 Universal (CC0 1.0) Public Domain Dedication \\
\hline
\end{tabular}

\subsection{Reification of n-ary relations to avoid blank nodes}

Blank nodes, i.e., resources without a URI, should be avoided when publishing LOD information [7], which poses a problem when $\mathrm{N}$-ary relations must be represented in the published dataset: the most straight forward way of representing $\mathrm{N}$-ary relations is by using blank nodes. ${ }^{5}$ This is the case for the OGOLOD dataset, since the relation between a gene and its GO term (location, function, or process) is qualified with an evidence code (e.g., a gene participates in a concrete process, and such relation is backed by an experimental confirmation). This problem was solved in OGOLOD by merging the OWL object properties with the evidence codes, i.e., expanding the properties according to the evidence codes: a subproperty of participates_in was generated for every evidence code, e.g., participatesEXP_in for Inferred from experiment, participates_IMP_in for Inferred from mutant phenotype, and so on. The same procedure was applied in the case of located_in.

\footnotetext{
${ }^{5}$ http://www.w3.org/TR/swbp-n-aryRelations/ \#anonvnamed
} 


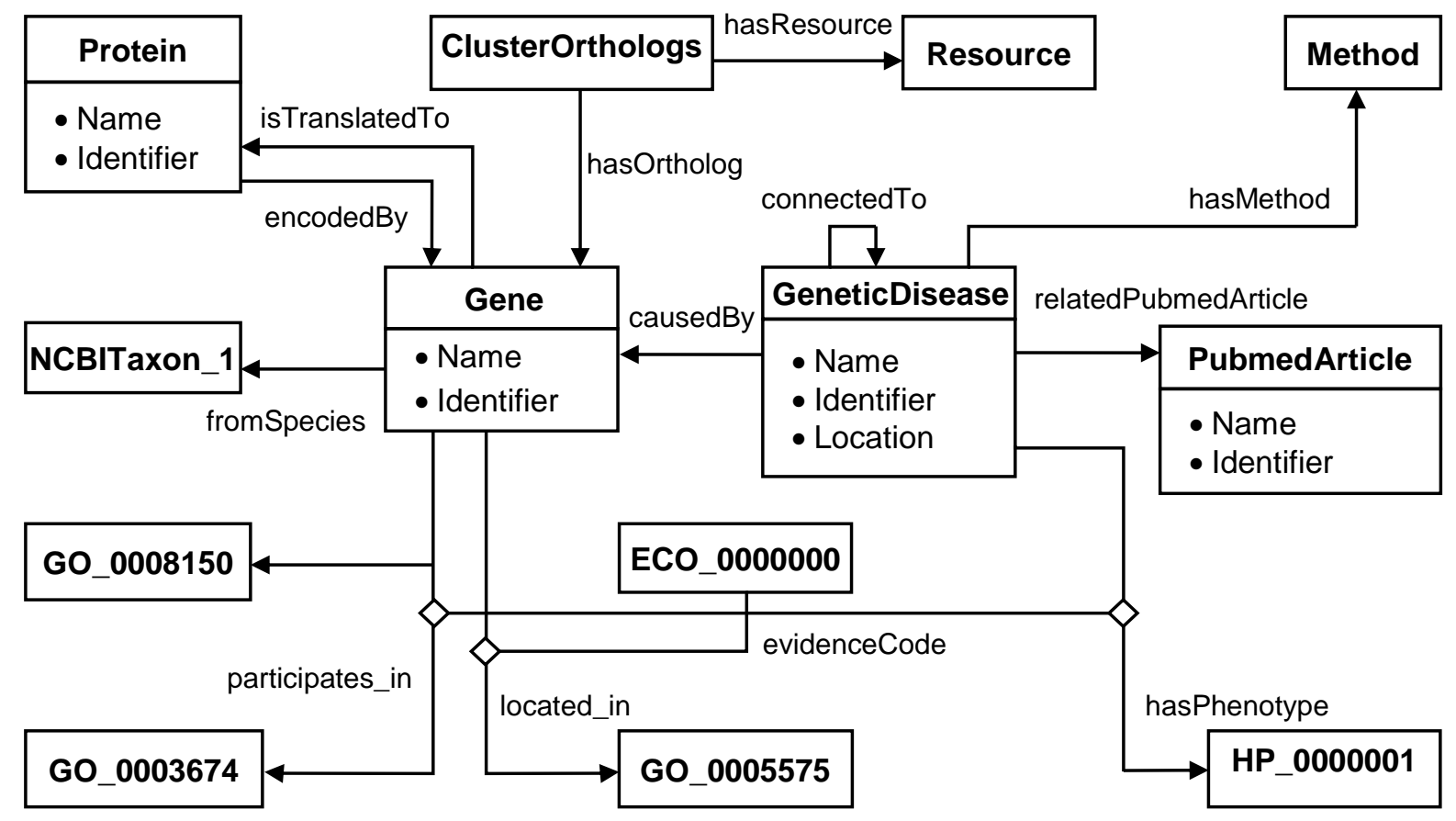

Fig. 1. OGO ontology. Figure reproduced from [10].

\subsection{Refactoring OWL Punning}

The original OGO KB exploited OWL punning ${ }^{6}$ in order to translate ontologies written in the $\mathrm{OBO}$ for$\mathrm{mat}^{7}$ to OWL. OBO terms were represented as entities in the OGO KB and depending on their particular use they can be considered as classes or instances but having the same URI. In other words, the same GO term is seen as a class when consulting their class hierarchy whereas it is seen as an instance when it is related to a particular gene through the relationships participates_in or located_in.

Therefore, the original OBO semantics is preserved but we are able to perform succinct SPARQL queries (without having to represent all the OWL axioms as RDF triples) ${ }^{8}$.

When using punning, entities can play different roles and they are differentiated at inference time by their axiomatic context. However, in a LOD setting this is not possible so any entity that exploits punning must

\footnotetext{
${ }^{6}$ http://www.w3.org/TR/owl2-new-features/ \#F12:_Punning

${ }^{7}$ http: //www. geneontology . org/Go. format. obo-1_2.shtml

${ }^{8}$ The general process of translating OBO ontologies to OWL using punning is described in detail in [2]
}

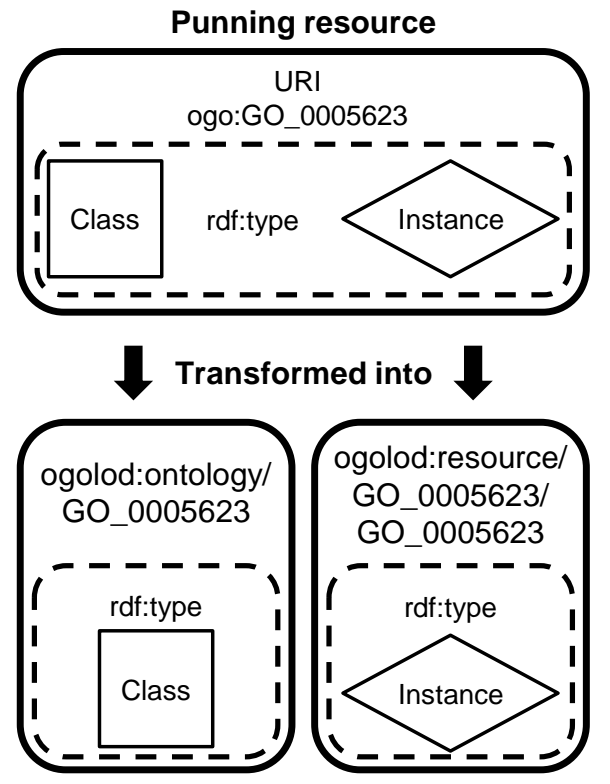

Fig. 2. Refactorisation of URIs of entities that exploit punning. Figure reproduced from [10].

be divided in the dataset into different entities (with different URIs) to represent every role. The entities were refactored by the process shown in Figure 2. 


\subsection{Linking to external datasets}

The binding process to link entities of the OGOLOD dataset to external datasets was manually defined. External datasets, such as bio2rdf, were examined to identify equivalent entities. The common properties and annotations between internal and external entities were manually identified to guide the binding process. Once the manual examination of the external datasets were completed, custom applications were developed to run this process. A more detailed description of the generation of OGOLOD dataset is provided in [10].

\subsection{Known shortcomings of the dataset}

The OGOLOD dataset is mainly composed of resources describing clusters of orthologous genes and genetic diseases, which were obtained from the OGO $\mathrm{KB}$. The links with external datasets were added during the translation process, so OGOLOD improves the OGO KB by increasing the number of external links to published datasets.

The OGOLOD dataset has injected new biomedical information that was not available to the LOD cloud. Consequently, the content of the OGOLOD dataset cannot be linked to the original source repositories, but to other datasets which publish resources describing genes and genetic diseases.

Life sciences is one of the most active disciplines in the publication of LOD datasets [7], thus there are many potentially linkable resources. However, the maintenance of high quality links to external datasets is a complex task due to the large size and diversity of such datasets. Therefore, methods to improve the management of such links are needed.

\subsection{Use cases}

The OGOLOD dataset relates orthologous genes from different species with human genetic disease descriptions. Orthology information is useful to produce predictions of gene function. Hence, obtaining disease information via clusters of orthologous genes can be used to find tentative gene functions.

As an example we propose the search for the human genetic diseases related to the gene Brcal of the Mus musculus species stored in bio2rdf.org datasets. This search is based on the content of OGOLOD and its external links associated with bio2rdf . org. Table 2 shows the SPARQL query associated to this example: in order to define the query the URL of the
Table 2

SPARQL query that relates genetic diseases and genes from bio2rdf.org.

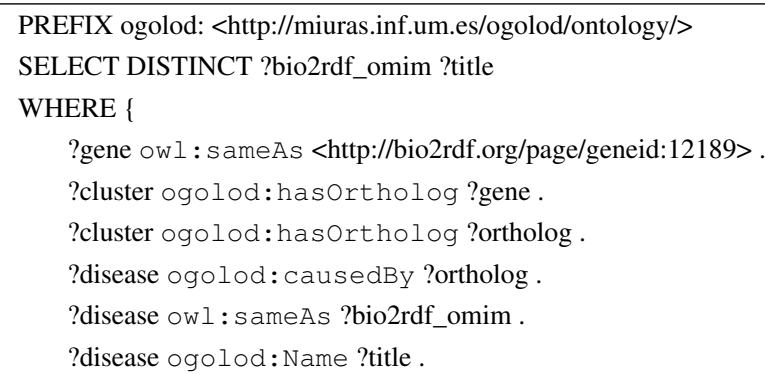

Table 3

Result of the SPARQL query from Table 2.

\begin{tabular}{|l|l|}
\hline ?bio2rdf_omim & http://bio2rdf.org/page/omim:113705 \\
\hline ?title & BREAST CANCER 1 GENE; BRCA1 \\
\hline
\end{tabular}

gene $^{9}$ is required as input. To reference the resources from bio2rdf . org we exploit the <owl : sameAs > relationships that relate OGOLOD resources with them.

Table 3 shows the result of the query from Table 2 . The resource from bio2rdf . rg $^{10}$ indicates the OMIM resource with identifier 113705 and it corresponds to breast cancer. Thus, we can link the Brcal gene from Mus musculus species with the Breast cancer disease resource, stored in separate bio2rdf . org datasets.

Table 4 provides another example in which orthologs related to genes involved in lung cancer and belonging to Rattus Norvegicus are retrieved. The result can be seen in 5 .

\section{Conclusion}

The OGOLOD dataset integrates information of orthologous genes and human genetic diseases in a LOD setting, making it computer-friendly and discoverable via other LOD links. OGOLOD can be used, for example, by scientists interested in studying diseases from a genetic perspective, using orthology information to infer new research paths for such disease. Providing the possibility of performing such analyses is OGOLOD's contribution to the LOD cloud, and more importantly, to translational biomedical research.

\footnotetext{
${ }^{9}$ http: //bio2rdf.org/page/geneid: 12189

${ }^{10}$ http://bio2rdf .org/page/omim:113705
} 
Table 4

SPARQL query that provides orthologous genes that belong to Rattus Norvegicus and are related to the genes involved in lung cancer

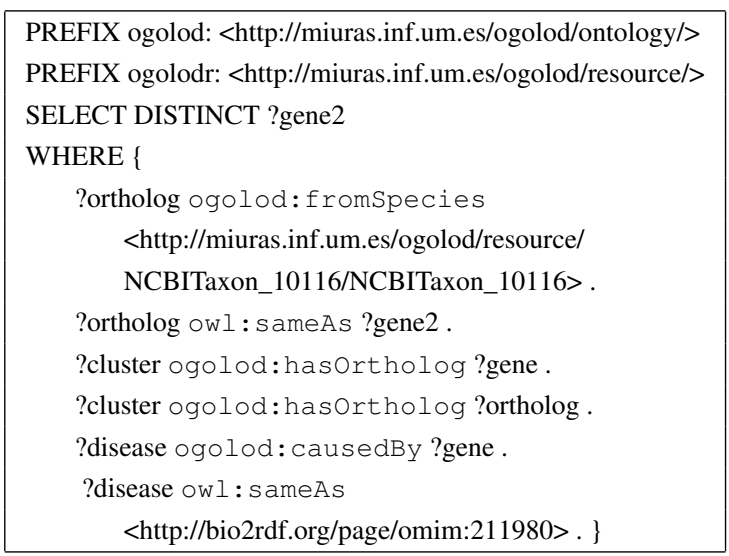

Table 5

Result of the SPARQL query from Table 4.

\begin{tabular}{|c|}
\hline gene2 \\
\hline http://bio2rdf.org/page/geneid:363140 \\
\hline
\end{tabular}

\section{Acknowledgements}

This work is funded by the Spanish Ministry for Economy and Competitiveness through grant TSI200766575-C02-02 and by the EC through the FEDER program. Mikel Egaña Aranguren is funded by the Marie Curie-COFUND Programme (FP7) of the European Commission. Boris Villazón-Terrazas is funded by the Web $n+1$ (TSI-020301-2009- 24) and BabelData (TIN2010-17550) projects.

\section{References}

[1] E. Antezana, M. Egaña, W. Blondé, A. Illarramendi, I. n. Bilbao, B. De Baets, R. Stevens, V. Mironov, and M. Kuiper. The Cell Cycle Ontology: An application ontology for the representation and integrated analysis of the cell cycle process. Genome Biology, 10(5):R58, 2009.

[2] M. E. Aranguren, J. T. F. Breis, E. Antezana, C. Mungall, A. R. González, and M. Wilkinson. OPPL-Galaxy, a Galaxy tool for enhancing ontology exploitation as part of bioinformatics workflows. Journal of Biomedical Semantics, 4(1):2+, 2013.

[3] F. Chen, A. Mackey, C. Stoeckert, and D. Roos. OrthoMCLDB: querying a comprehensive multi-species collection of ortholog groups. Nucleic Acids Res, 34(Suppl 1):D363-D368, 2006.
[4] C. Dessimoz, T. Gabaldón, D. S. Roos, E. L. L. Sonnhammer, J. Herrero, and the Quest for Orthologs Consortium. Toward community standards in the quest for orthologs. Bioinformatics, 28(6):900-904, 2012.

[5] Gene Ontology Consortium. Gene Ontology: tool for the unification of biology. Nature Genetics, 23(May):25-29, 2000.

[6] K. Harvey, C. Pfleger, and I. Hariharan. The Drosophila Mst ortholog, hippo, restricts growth and cell proliferation and promotes apoptosis. Cell, 114:457-467, August 2003.

[7] T. Heath and C. Bizer. Linked Data: Evolving the Web into a Global Data Space. Morgan \& Claypool, 2011.

[8] J. A. Miñarro Gimenez, M. Egaña Aranguren, R. Martínez Béjar, J. T. Fernández-Breis, and M. Madrid. Semantic integration of information about orthologs and diseases: The OGO system. Journal of biomedical informatics, (44):1020-1031, 2011.

[9] J. A. Miñarro Gimenez, M. Madrid, and J. T. Fernandez-Breis. OGO: an ontological approach for integrating knowledge about orthology. BMC bioinformatics, 10 Suppl 10(Suppl 10):S13+, 2009.

[10] J. A. Miñarro-Gimenez, M. E. Aranguren, B. V. Terrazas, and J. T. Fernández-Breis. Publishing Orthology and Diseases Information in the Linked Open Data cloud. Current Bioinformatics, 7(3):255-266, 2012.

[11] M. Remm, C. V. Storm, and E. L. Sonnhammer. Automatic clustering of orthologs and in-paralogs from pairwise species comparisons. Journal of Molecular Biology, 314(5):10411052, 2001.

[12] P. Robinson, S. Köhler, S. Bauer, D. Seelow, D. Horn, and S. Mundlos. The Human Phenotype Ontology: a tool for annotating and analyzing human hereditary disease. The American Journal of Human Genetics, 83:610-615, 2008.

[13] E. Sayers, T. Barrett, D. Benson, E. Bolton, S. Stephen, K. Canese, V. Chetvernin, D. Church, M. Deanna, M. DiCuccio, S. Federhen, M. Feolo, L. Y. L.Y.Geer, W. Helmberg, Y. Kapustin, D. Landsman, D. J. Lipman, Z. Lu, T. L. Madden, T. Madej, D. R. Maglott, A. Marchler-Bauer, V. Miller, I. Mizrachi, J. Ostell, A. Panchenko, K. D. Pruitt, G. D. Schuler, E. Sequeira, S. T. Sherry, M. Shumway, K. Sirotkin, D. Slotta, A. Souvorov, G. Starchenko, T. A. Tatusova, L. Wagner, Y. Wang, W. John Wilbur, E. Yaschenko, and J. Ye. Database resources of the National Center for Biotechnology Information. Nucleic Acids Research, 38(suppl 1):D5-D16, 2010.

[14] B. Smith, W. Ceusters, B. Klagges, J. Kohler, A. Kumar, J. Lomax, C. Mungall, F. Neuhaus, A. Rector, and C. Rosse. Relations in Biomedical Ontologies. Genome Biology, 6:R46, 2005.

[15] R. Tatusov, N. Fedorova, J. Jackson, A. Jacobs, B. Kiryutin, E. Koonin, D. Krylov, R. Mazumder, S. Mekhedov, A. Nikolskaya, S. Rao, S. Smirnov, A. Sverdlov, S. Vasudevan, Y. Wolf, J. Yin, and D. Natale. The COG database: an updated version includes eukaryotes. BMC Bioinformatics, 4:41-55, 2003.

[16] B. Villazón-Terrazas, L. Vilches-Blázquez, O. Corcho, and A. Gómez-Pérez. Methodological Guidelines for Publishing Government Linked Data Linking Government Data. In D. Wood, editor, Linking Government Data, chapter 2, pages 27-49. Springer New York, 2011. 